\title{
Dyskinesien und Fluktuationen in den Griff bekommen
}

\author{
Bei fortgeschrittener Parkinson-Erkrankung ist die \\ L-Dopa-Therapie durch motorische Fluktuationen \\ wie Wearing-off- oder On-/Off-Phänomene und \\ Dyskinesien limitiert. Vor allem die Fluktuationen \\ der Wirksamkeit werden von den Patienten als \\ besonders belastend empfunden.
}

In randomisierten kontrollierten Studien entwickeln $16 \%$ der Patienten bereits nach neun Monaten L-Dopa-Therapie motorische Komplikationen, nach zwei Jahren sind es bereits $30-40 \%$. Patienten, die bereits in jungen Jahren an Parkinson erkranken („Young-onset-Parkinson“) sind besonders stark betroffen, hier liegt die Rate nach fünf Jahren bei $90 \%$, sagte Paolo Barone aus Napoli, Italien.

In einer Befragung von 172 Patienten mit fortgeschrittener Parkinson-Erkrankung wurden die Wirksamkeitsfluktuationen als das am stärkten belastende Symptom bewertet. Viele adjuvante Therapien zielen daher darauf ab, die motorischen Fluktuationen durch eine verlängerte L-Dopa-Wirkung zu reduzieren. Als effektiv haben sich dabei der MAO-Hemmer Rasagilin, der COMT-Inhibitor Entacapone, Dopaminagonisten (Ropinirol, Pramipexol, Rotigotin) und Safinamid (Hemmung MAO-B und Glutamat-Modulation) erwiesen. Auch die ApomorphinPumpen-Therapie ist eine Option bei Dyskinesien und Fluktuationen. In vielen Studien konnte hier eine Reduktion der OffZeit und eine Einsparung von L-Dopa gezeigt werden.

Je höher die L-Dopa-Dosis, umso größer ist das Risiko für die Entwicklung von Dyskinesien und Wearing-off-Phänomen. Bei Dosen $<400$ mg/Tag sind nach 182 Wochen $12,1 \%$ von Dyskinesien betroffen, bei Dosen über $600 \mathrm{mg} / \mathrm{Tag}$ 55,8 \% der Patienten. Ein ähnlicher Unterschied zeigt sich bei Wearing-off-Phänomenen (27,2 \% und 72,6\%). Die Patienten sollten daher so lange wie möglich niedrige L-Dopa-Dosen erhalten, sagte Barone.

Gerade Wearing-off-Phänomene werden zu Beginn der Erkrankung von Ärzten oft nicht so gut wahrgenommen, sagte der Neurologe. So berichten innerhalb der ersten zweieinhalb Jahre $42 \%$ der Patienten von Fluktuationen, aber nur $22 \%$ der Ärzte sehen solche Phänomene bei ihren Patienten. Beim Erkennen nicht motorischer Fluktuationen wie Angst falle das Ergebnis wahrscheinlich noch schlechter aus, meinte Barone.

Maria Weiß

Symposium "Customizing Treatment of Movement Disorders Responsive to Dopaminerg Therapy", 20. International Congress of Parkinson's Disease and Movement Disorders, 20.6.2016, Berlin

\section{THS - nicht alle Symptome sprechen gleichermaßen gut an}

\begin{abstract}
Die tiefe Hirnstimulation (THS) stellt für Patienten mit idiopathischem Parkinson-Syndrom, die nicht mehr ausreichend auf L-Dopa ansprechen, eine mögliche Therapieoption dar. Das Zielgebiet der Neurostimulation sollte dabei nach der vorherrschenden Symptomatik gewählt werden.
\end{abstract}

Traditionelle Kandidaten für eine THS sind Patienten mit idiopathischem Parkinson-Syndrom, die trotz kontinuierlichen Ansprechens auf L-Dopa mit den üblichen Medikamenten nicht mehr adäquat behandelt werden können und eine signifikante Behinderung aufweisen. Außerdem wird gefordert, dass keine signifikante Demenz oder Depression besteht und keine chirurgischen Risikofaktoren vorliegen. Die Patienten sollten zudem realistische Erwartungen haben und sich keine „Heilung“ der Erkrankung versprechen, sagte Dr. Jill Ostrem aus Greenbrae, Kalifornien.

Nicht alle Symptome sprechen gleichermaßen auf die THS an. Gute Erfolge werden bei On-/Off-Fluktuationen, Dyskinesien, Tremor, Rigidität, Bradykinesie und schlurfendem Gang erzielt, manchmal auch bei Freezing. Eher keine Verbesserungen wurden dagegen bei Sprach- und Schluckstörungen, Gleichgewichtsstörungen, Freezing des Gangs unter Medikation und bei den meisten nicht motorischen Symptomen festgestellt.

Entscheidend für den therapeutischen Erfolg könnte das Zielgebiet der Neurostimulation sein. Das zuerst eingesetzte Ziel- gebiet war der Nucleus ventralis intermedius (VIM) des Thalamus. Hier wirkt die THS sehr gut gegen den Tremor, zeigt aber nur minimale Effekte auf andere Symptome wie Rigidität, Bradykinesie, axiale Symptome und Dyskinesien. Eine Option ist dieses Verfahren auch heute noch bei älteren stabilen Patienten mit Tremor als führendem Symptom, so Ostrem.

Weitere mögliche Zielregionen sind der Nucleus subthalmicus (STN) und der Globus pallidus interna. Beide Verfahren wirken ähnlich gut auf motorische Symptome wie Rigidität, Bradykinesie, Tremor sowie die Lebensqualität, wobei die STNStimulation häufiger eine Reduktion der Medikamente zu ermöglichen scheint. Auf der anderen Seite kann die STN-Stimulation auch Dyskinesien stimulieren, sodass man hier bei jedem Patienten individuell abwägen muss, sagte die Neurologin.

Wie sieht es mit nicht motorischen Symptomen aus? Kognitive Funktionen, Verhaltensstörungen und Depressionen werden durch THS in der Regel nicht verbessert und können sogar schlechter werden. Bei einigen Symptomen wie Miktionsstörungen, Obstipation und Schmerz sind zum Teil leichte Verbesserungen beschrieben. Hier müsse in Zukunft möglicherweise nach neuen Biomarkern für ein Ansprechen oder nach neuen Zielgebieten für die THS gesucht werden.

Maria Weiß

Symposium "Customizing Treatment of Movement Disorders Responsive to Dopaminerg Therapy", 20. International Congress of Parkinson's Disease and Movement Disorders, 20.6.2016, Berlin 